\title{
Probing strong dynamics with cosmic neutrinos
}

\author{
Luis A. Anchordoqui, ${ }^{1,2,3}$ Carlos García Canal, ${ }^{4}$ and Jorge F. Soriano ${ }^{1}$ \\ ${ }^{1}$ Department of Physics and Astronomy, Lehman College, \\ City University of New York, New York 10468, USA \\ ${ }^{2}$ Department of Physics, Graduate Center, City University of New York, New York 10016, USA \\ ${ }^{3}$ Department of Astrophysics, American Museum of Natural History, New York 10024, USA \\ ${ }^{4}$ Instituto de Física La Plata, UNLP, CONICET Departamento de Física, Facultad de Ciencias Exactas, \\ Universidad Nacional de La Plata, C.C. 69, (1900) La Plata, Argentina
}

(Received 2 March 2019; published 1 November 2019)

\begin{abstract}
IceCube has observed 80 astrophysical neutrino candidates in the energy range $0.02 \lesssim E_{\nu} / \mathrm{PeV} \lesssim 2$. Deep inelastic scattering of these neutrinos with nucleons on Antarctic ice sheet probe center-of-mass energies $\sqrt{s} \sim 1 \mathrm{TeV}$. By comparing the rates for two classes of observable events, any departure from the benchmark (perturbative QCD) neutrino-nucleon cross section can be constrained. Using the projected sensitivity of South Pole next-generation neutrino telescope we show that this facility will provide a unique probe of strong interaction dynamics. In particular, we demonstrate that the high-energy high-statistics data sample to be recorded by IceCube-Gen2 in the very near future will deliver a direct measurement of the neutrino-nucleon cross section at $\sqrt{s} \sim 1 \mathrm{TeV}$, with a precision comparable to perturbative QCD informed by HERA data. We also use IceCube data to extract the neutrino-nucleon cross section at $\sqrt{s} \sim 1 \mathrm{TeV}$ through a likelihood analysis, considering (for the first time) both the charged-current and neutral-current contributions as free parameters of the likelihood function.
\end{abstract}

DOI: 10.1103/PhysRevD.100.103001

\section{INTRODUCTION}

High-energy neutrinos are unique messengers of faraway phenomena and can serve as a probe of new physics at sub-fermi distances. Per contra the promise of high energy neutrinos might appear to be severely limited by astrophysical uncertainties. Event rates constrain only a combination of fluxes and cross sections, and so astrophysical uncertainties cloud particle physics implications and vice versa. However, the event rates for up- and downgoing neutrinos depend differently on neutrino cross sections $[1,2]$. By combining both up- and downgoing data one may therefore disentangle particle physics from astrophysics and constrain both the properties of astrophysical sources and neutrino interactions. This technique is entirely agnostic to any physics process which may modify the neutrino-nucleon cross section. Essentially this approach constitutes a straightforward counting experiment.

In this paper we adopt this technique to investigate the sensitivity of future South Pole neutrino-detectionexperiments to the neutrino-nucleon cross section.

Published by the American Physical Society under the terms of the Creative Commons Attribution 4.0 International license. Further distribution of this work must maintain attribution to the author(s) and the published article's title, journal citation, and DOI. Funded by SCOAP ${ }^{3}$.
Earlier work in this area has generally assumed a plausible neutrino luminosity [1-7]. Now, however, IceCube measurements yield a nonzero neutrino event rate at $\mathrm{PeV}$ energies [8-13], allowing for a more reliable calculation. Indeed, the IceCube Collaboration recently reported a measurement of the neutrino-nucleon cross section [14]. For neutrinos in the energy bin $6.3<E_{\nu} / \mathrm{TeV}<980$, the measured cross section is

$$
\sigma_{\nu N}=\sigma_{\mathrm{SM}} \times\left[1.30_{-0.19}^{+0.21}(\mathrm{stat})_{-0.43}^{+0.39}(\mathrm{syst})\right]
$$

where $\sigma_{\mathrm{SM}}$ is the Standard Model (SM) prediction $[15,16]$. Further analysis of the IceCube data-sample allowed determination of the energy dependence of the cross section [17]. The proposed IceCube-Gen2 [18] will surely perform technologically at least at the level of IceCube, so a conservative estimate of the sample size is attainable by simply scaling the aperture. IceCube-Gen2 will have an order of magnitude larger aperture than IceCube, which should provide a sample large enough for a precision measurement of the neutrion-nucleon cross section. Indeed as we show herein IceCube-Gen2 will be able to determine the neutrino-nucleon cross section with a precision comparable to perturbative QCD informed by collider data.

The layout of the paper is as follows. In Sec. II we provide an overview of neutrino detection at IceCube and describe the different event topologies. After that we infer 
the sensitivity of IceCube to the neutrino-nucleon interaction cross section by combining upward- and downwardgoing event rates. In Sec. III we describe the particulars of our likelihood approach and present the results from data analysis. We begin by making use of the high-energy reach of IceCube data to extract the neutrino-nucleon cross section at energies beyond those available in manmade neutrino beams. As in previous studies [14,17], we test strong dynamics by fixing the ratio of charged to neutral current processes to that of the perturbative SM. To test nonperturbative SM phenomena, herein we also consider the ratio of charged to neutral current processes to be a free parameter of the likelihood function. Then, armed with our findings, we investigate the sensitivity of future South Pole neutrino-detection-experiments to the neutrino-nucleon cross section. Our conclusions are collected in Sec. IV.

Before proceeding, it is important to stress that for neutrino energies $\lesssim 10 \mathrm{PeV}$, perturbative QCD provides a robust framework to calculate the neutrino-nucleon cross section [19-24]. It is only when the fractional momenta $x$ carried by the constituents become vanishingly small that the structure functions develop a $\ln (1 / x)$ divergent behavior, which in turn results in a violation of unitarity bounds. Consequently, perturbative QCD predictions are expected to break down solely when the nucleon has an increasing number of partons with small $x$. For the center of mass energies relevant to our study, however, the neutrinonucleon cross section can be calculated perturbatively with an accuracy of better than 5\% when constrained by measured HERA structure functions [15,16]. Though HERA measurements have significantly bounded the behavior of neutrino scattering for $E_{\nu} \lesssim 10 \mathrm{PeV}$, we note that the analysis discussed herein provides an independent direct measurement of the neutrino-nucleon cross section in this energy range, and hence is complementary to the laboratory results.

\section{NEUTRINO INTERACTIONS AT ICECUBE}

Neutrino (antineutrino) interactions in the Antarctic ice sheet can be reduced to two categories: (i) in charged current (CC) interactions the neutrino becomes a charged lepton through the exchange of a $W^{ \pm}$with some nucleon $N$, $\nu_{\alpha}\left(\bar{\nu}_{\alpha}\right)+N \rightarrow \ell_{\alpha}^{ \pm}+$anything; (ii) in neutral current (NC) interactions the neutrino interacts via a $Z$ transferring momentum to jets of hadrons, but producing a neutrino rather than a $\ell^{ \pm}$in the final state: $\nu_{\alpha}\left(\bar{\nu}_{\alpha}\right)+N \rightarrow \nu_{\alpha}\left(\bar{\nu}_{\alpha}\right)+$ anything. Lepton flavor is labeled as $\alpha \in\{e, \mu, \tau\}$ from here on.

The three neutrino species engender distinctive signal morphologies when they interact in ice producing the Cherenkov light detected by the IceCube digital optical modules (DOM), each with a ten-inch photomultiplier tube (PMT) and associated electronics. The CC interaction of $\nu_{e}$ triggers an electromagnetic cascade (or shower) which ranges out quickly. Such a cascade produces a rather
TABLE I. Event topology for each neutrino flavor.

\begin{tabular}{llll}
\hline \hline Interaction type & $e$ & $\mu$ & $\tau$ \\
\hline CC & $\mathcal{S}$ & $\mathcal{T}$ & $\mathcal{S}$ \\
NC & $\mathcal{S}$ & $\mathcal{S}$ & $\mathcal{S}$ \\
\hline \hline
\end{tabular}

spherically symmetric signal, and therefore exhibits a low angular resolution of about $15^{\circ}-20^{\circ}$ [9]. However, a fully or mostly contained shower event provides a relatively precise measurement of the $\nu_{e}$ energy, with a resolution of $\Delta\left(\log _{10} E_{\nu}\right) \approx 0.26$ [25]. The situation is reversed for $\mathrm{CC}$ interaction $\nu_{\mu}$ induced events. In this case, the secondary muon travels relatively unscathed through the ice leaving behind a track. Muon tracks point nearly in the direction of the original $\nu_{\mu}$, allowing one to infer the arrival direction with high angular resolution (say $\sim 0.7^{\circ}$ ), while the electromagnetic equivalent energy deposited $E_{\text {dep }}$ represents only a lower bound of the genuine $\nu_{\mu}$ energy. For muon tracks, we adopt estimates derived elsewhere [26] and set the fractional energy $E_{\mu}^{\mathrm{dep}} / E_{\nu}$ to $0.57,0.51,0.50$, and 0.47 for the IceCube data set in the interval 10$100 \mathrm{TeV}, 100-200 \mathrm{TeV}, 200 \mathrm{TeV}-1 \mathrm{PeV}$, and 1-10 PeV; respectively. A point worth noting at this juncture is that the probability distributions for the parent neutrino energy of a muon track event which deposits an energy $E_{\mu}^{\text {dep }}$ shown in Fig. 1 of Ref. [27] are in good agreement with the estimates of $E_{\mu}^{\text {dep }} / E_{\nu}$ adopted herein. Lastly, $\nu_{\tau} \mathrm{CC}$ interactions may, depending on the neutrino energy, produce double bang events [28], with one shower produced by the initial $\nu_{\tau}$ collision in the ice, and the second shower resulting from most subsequent $\tau$ decays. Separation of the two bangs is only feasible for $E_{\nu}>3 \mathrm{PeV}$, whereas at lower energies the showers tend to overlap one another. NC interactions of all $\nu$ flavors also produce showers, but with a smaller rate than $\mathrm{CC}$ interactions. For the energy range of interest, there are two different topologies for the events registered at IceCube, namely tracks $(\mathcal{T})$ and showers $(\mathcal{S})$. ${ }^{1}$ Each of them is produced by different neutrino flavors and interactions, as summarized in Table I.

The classification of observed events in different topologies is not always straightforward. While almost all NC $\nu_{\mu}$ events are generally correctly classified as showers, a non negligible number of $\mathrm{CC} \nu_{\mu}$ events, of both atmospheric and astrophysical origin, could be misclassified as showers if the muon has too little energy or is produced near the edge of the detector, escaping in both cases without enough

\footnotetext{
${ }^{1}$ We note in passing that the flavor of a $\mathrm{CC} \nu_{\tau}$ interaction of $\mathcal{S}$ topology (i.e., in which the two bangs cannot be separately reconstructed) can be identified by searching for double pulse waveforms that are consistent with $\nu_{\tau} \mathrm{CC}$ interaction signatures in IceCube, while rejecting waveforms with features that are consistent with late scattered photons from single cascade events from $\mathrm{NC}$ and $\nu_{e} \mathrm{CC}$ interactions [29].
} 
energy deposited to be detected [11,30]. The effects of these misclassifications have been studied in great detail in Ref. [31-33]. While accounting for misclassifications increases the fraction of $\mu$-neutrinos and may have influence on the flavor ratios, with present statistics it does not influence neither the shape of the spectrum for a shower plus track analysis [32] nor cross section studies. In light of this, we assume here the event topologies of IceCube highenergy starting events (HESE) at face value as given in $[9,12,13]$.

The rates at IceCube for down- and up-going events have been found [34] to scale respectively as $\Gamma_{\text {down }} \propto \phi \sigma_{i}$ and $\Gamma_{\text {up }} \propto \phi \sigma_{i} / \sigma_{a}$, where $\phi$ is the neutrino flux, $\sigma_{i}$ is the cross section for the interaction that produces the event $(i \in\{\mathrm{CC}, \mathrm{NC}\})$, and $\sigma_{a}$ is the attenuation cross section, which includes all the effects decreasing the luminosity due to the fact that neutrinos have to traverse the Earth; see the Appendix A for details.

For a given bin of energy, we can constrain neutrino interactions without assuming particular neutrino fluxes or cross sections. It will be convenient, however, to present results relative to standard reference values. IceCube data are consistent with isotropic arrival directions [35] and with expectations for equal fluxes of all three neutrino flavors [30-33]. For the reference flux, we adopt the central value of the best-fit power law of the $4 \mathrm{yr}$ IceCube data [10],

$\phi_{0}\left(E_{\nu}\right)=2.2 \times 10^{-18}\left(\frac{E_{\nu}}{100 \mathrm{TeV}}\right)^{-2.58}\left(\mathrm{Gevssrcm}^{2}\right)^{-1}$,

per flavor $\nu_{\alpha}+\bar{\nu}_{\alpha}$. For the reference cross sections, we choose the results from perturbative QCD calculations constrained by HERAPDF1.5 shown in Fig. 1. These cross sections have been the benchmarks adopted by the IceCube Collaboration [14].

For a given flux $\phi$ and cross sections $\sigma_{i}$ and $\sigma_{a}$, the expected number of upgoing events of a flavor $\alpha$ produced by a charged or neutral current interaction may be expressed as

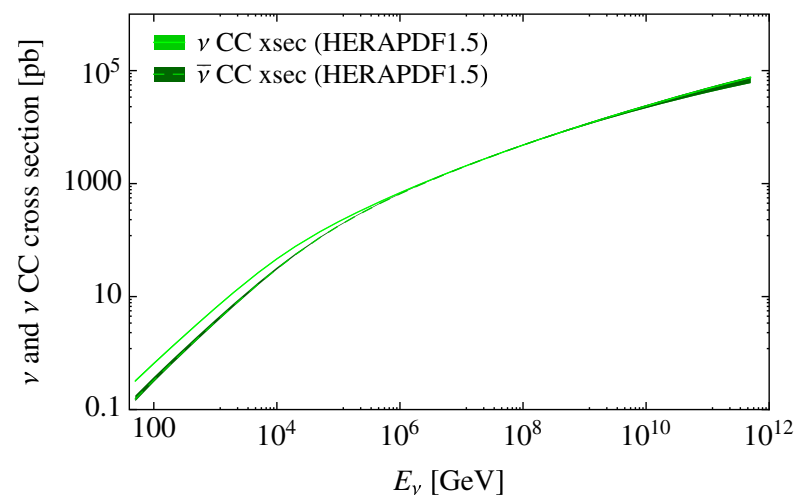

$$
N_{u}^{i, \alpha} \equiv \tilde{N}_{u}^{i, \alpha} \frac{\phi}{\phi_{0}} \frac{\sigma_{i} / \sigma_{a}^{\alpha}}{\sigma_{i, 0} / \sigma_{a, 0}^{\alpha}}
$$

and for downgoing events,

$$
N_{d}^{i, \alpha} \equiv \tilde{N}_{d}^{i, \alpha} \frac{\phi}{\phi_{0}} \frac{\sigma_{i}}{\sigma_{i, 0}}
$$

with $i \in\{\mathrm{CC}, \mathrm{NC}\}$ and where the $\tilde{N}$-constants are obtained assuming that the flux and cross sections are equal to the reference values, $\sigma_{i, 0}$ and $\sigma_{a, 0}$.

At this stage it is worthwhile to point out that we have $12 N$ quantities ( 2 directions $\times 2$ interactions $\times 3$ flavors), but only 4 of them will be considered in the data analysis ( 2 topologies $\times 2$ directions). To gather the events adequately we define the four quantities

$$
N_{x}^{\mathcal{Z}} \equiv \sum_{(i, \alpha) \in \mathcal{Z}} N_{x}^{i, \alpha},
$$

with $x \in\{u, d\}$ referring to up- or downgoing events, and $\mathcal{Z} \in\{\mathcal{T}, \mathcal{S}\}$ referring to the event topology (track or shower, respectively). The sum is extended to the pairs $(i, \alpha)$ contributing to a topology $\mathcal{Z}$, according to Table I.

We define $\phi \equiv F \phi_{0}, \sigma_{\mathrm{tot}} \equiv S \sigma_{\mathrm{tot}, 0}$ and the partial cross sections $\sigma_{i, 0} \equiv \alpha_{i, 0} \sigma_{\mathrm{tot}, 0}$ and $\sigma_{i} \equiv \alpha_{i} \sigma_{\mathrm{tot}}$. The flavor dependent attenuation cross sections are expressed as $\sigma_{a, 0}^{\alpha} \equiv$ $a_{\alpha, 0} \sigma_{\mathrm{tot}, 0}$ and $\sigma_{a}^{\alpha} \equiv a_{\alpha} \sigma_{\mathrm{tot}}$. The $a$ constants may be expressed in terms of the interaction inelasticities and the $\alpha$ parameters as $a_{\alpha, 0}=\sum_{i} y_{i, 0}^{\alpha} \alpha_{i, 0}$ and $a_{\alpha}=\sum_{i} y_{i}^{\alpha} \alpha_{i}$, where $i$ refers to $\mathrm{CC}$ or NC, and $y_{i}^{\alpha}$ are the inelasticity parameters for each interaction [19]. We can now rewrite (4) as

$$
\begin{aligned}
& N_{d}^{\mathcal{Z}}=F S \sum_{(i, \alpha) \in \mathcal{Z}} \frac{\alpha_{i}}{\alpha_{i, 0}} \tilde{N}_{d}^{i, \alpha}, \\
& N_{u}^{\mathcal{Z}}=F \sum_{(i, \alpha) \in \mathcal{Z}} \frac{a_{\alpha, 0} \alpha_{i}}{a_{\alpha} \alpha_{i, 0}} \tilde{N}_{u}^{i, \alpha} .
\end{aligned}
$$

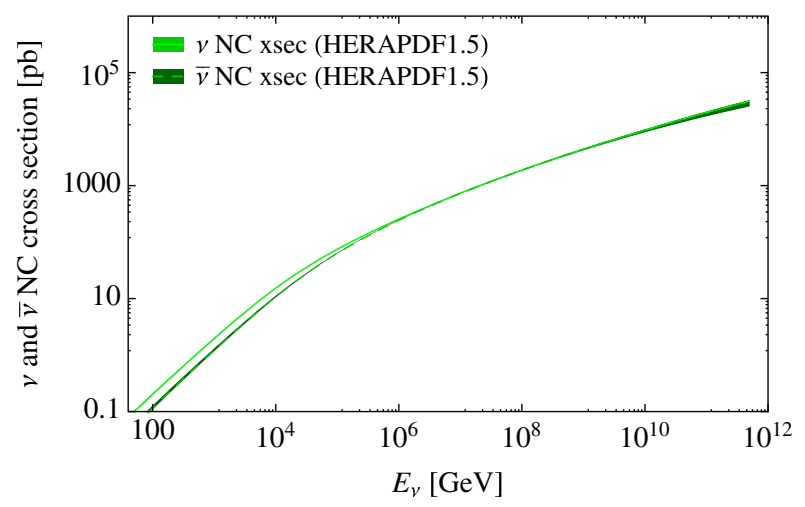

FIG. 1. Neutrino and antineutrino cross sections on isoscalar targets for $\mathrm{CC}$ and $\mathrm{NC}$ scattering according to HERAPDF1.5; $\sigma_{\mathrm{CC}, 0}$ and $\sigma_{\mathrm{NC}, 0}$, respectively. Taken from Ref. [15]. 
To perform any further analysis we need to calculate the reference number of events $\left(\tilde{N}_{x}^{i, \alpha}\right)$ obtained for the flux $\phi_{0}$ and cross sections $\sigma_{i, 0}$ and $\sigma_{a, 0}$ for each of the 12 quantities involved in (5). This can be done by means of the expression

$$
\tilde{N}_{x}^{i, \alpha} \equiv 2 \pi T \int_{E_{\min }}^{E_{\max }} \phi_{0}\left(E_{\nu}\right) A_{x}^{i, \alpha}\left(E_{\nu}\right) \mathrm{d} E_{\nu}
$$

where $T$ is the running time of the experiment for this sample and $A_{x}^{i, \alpha}$ is the effective area averaged for up-(northern) or down-(southern) going (hemisphere) neutrinos per flavor $\alpha$ producing an event after a $i$-type interaction. From the IceCube effective area reported in [9], we obtain the quantity $A_{x}^{\mathrm{CC}, \alpha}+A_{x}^{\mathrm{NC}, \alpha}$. To isolate the interaction dependence we introduce the weights

$$
w^{i, \alpha} \equiv \frac{\sigma_{i} M_{i}^{\alpha}}{\sum_{k} \sigma_{k} M_{k}^{\alpha}}=\frac{\alpha_{i} M_{i}^{\alpha}}{\sum_{k} \alpha_{k} M_{k}^{\alpha}},
$$

where $M_{i}^{\alpha}$ is the IceCube target mass for flavor $\alpha$ and interaction type $i$, given also in [9]. It follows that

$$
\tilde{N}_{x}^{i, \alpha}=w^{i, \alpha} \tilde{N}_{x}^{\alpha},
$$

and so

$$
\tilde{N}_{x}^{\alpha} \equiv 2 \pi T \int_{E_{\min }}^{E_{\max }} \phi_{0}\left(E_{\nu}\right) A_{x}^{\alpha}\left(E_{\nu}\right) \mathrm{d} E_{\nu}
$$

The events are distributed in the same energy bins used in $[9,12,13]$. For the $k$ th bin, containing events in the energy range $\left[E_{\min }^{k}, E_{\max }^{k}\right)$, we use in (9) the bin averaged effective area $\left\langle A_{x}^{\alpha}\right\rangle_{k}$ from [9], and the flux per flavor given in (2). This gives us the reference values in each bin as

$$
\tilde{N}_{x, k}^{\alpha} \equiv 2 \pi T\left\langle A_{x}^{\alpha}\right\rangle_{k} \int_{E_{\min }^{k}}^{E_{\max }^{k}} \phi\left(E_{\nu}\right) \mathrm{d} E_{\nu} .
$$

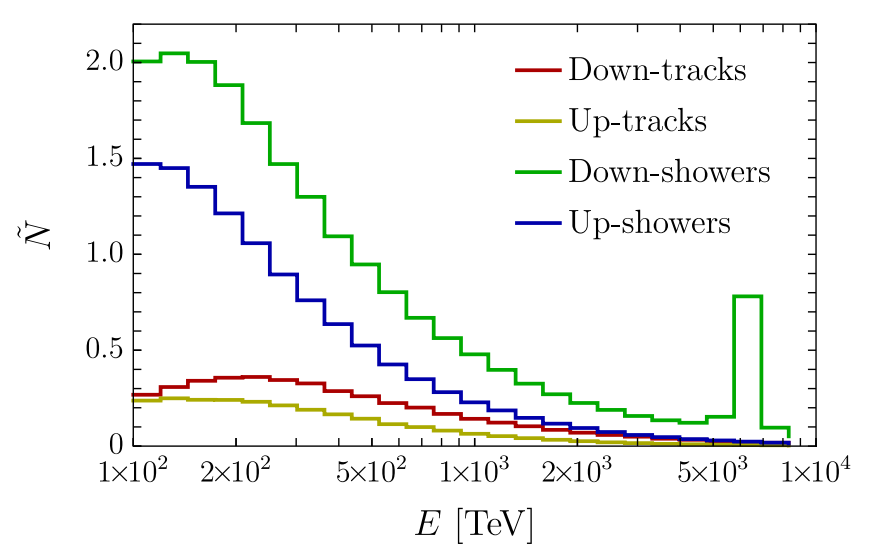

FIG. 2. Reference number of events from (10).
The values of the expected number of events are shown in Fig. 2.

In 6 years of observation IceCube has detected above about 30 neutrino events with energies in the range $0.1<E_{\nu} / \mathrm{PeV}<2$. This implies that in 10 years of data taken this facility will collect on the order of 50 neutrino events within this decade of energy. The next-generation of neutrino telescope in the South pole, IceCube-Gen2, will increase the per year exposure by about an order of magnitude, and therefore in $10 \mathrm{yr}$ of observation will collect roughly 500 neutrinos with $0.1<E_{\nu} / \mathrm{PeV}<2$.

In the next section we generalize the full-likelihood approach introduced in [26] to disentangle cross section parameters in (9) from flux uncertainties in the IceCube data sample.

\section{LIKELIHOOD ANALYSIS}

Armed with IceCube observations and expected event rates for fiducial flux and cross sections we now perform the analysis to extract cross section parameters using a maximum likelihood method. Let $\boldsymbol{\theta}$ be the set of parameters involved in the data analysis, containing $F$ and all the relevant guidelines to vary the $\sigma_{\mathrm{CC}, 0}$ and $\sigma_{\mathrm{NC}, 0}$ cross sections. Let $\bar{N}_{x, k}^{Z}$ be the measured number of events with topology $\mathcal{Z} \in\{\mathcal{S}, \mathcal{T}\}$ and direction $x \in\{u, d\}$ in the energy bin $k$. The probability that the bin $k$ contains $\bar{N}_{x, k}^{\mathcal{Z}}$ events of type $(x, \mathcal{Z})$ while expecting $N_{x, k}^{\mathcal{Z}}(\boldsymbol{\theta})$ is given by a Poisson distribution

$$
f\left[\bar{N}_{x, k}^{\mathcal{Z}} \mid N_{x, k}^{\mathcal{Z}}(\boldsymbol{\theta})\right]=\frac{e^{-N_{x, k}^{\mathcal{Z}}}\left(N_{x, k}^{\mathcal{Z}}\right)^{\bar{N}_{x, k}^{\mathcal{Z}}}}{\bar{N}_{x, k}^{\mathcal{Z}} !},
$$

while the probability that the bin $k$ contains $\bar{N}_{x, k}^{\mathcal{Z}}$ events of type $(x, \mathcal{Z})$ for all the types is

$$
\mathcal{F}_{k}(\boldsymbol{\theta}) \equiv \prod_{x, \mathcal{Z}} f\left[\bar{N}_{x, k}^{\mathcal{Z}} \mid N_{x, k}^{\mathcal{Z}}(\boldsymbol{\theta})\right]
$$

The likelihood of having a given a set of parameters $\boldsymbol{\theta}$ observing the actual event distribution is

$$
\mathcal{L}(\boldsymbol{\theta})=\prod_{k} \mathcal{F}_{k}(\theta)
$$

By the maximization of $\mathcal{L}$ in terms of the parameters $\boldsymbol{\theta}$ we will estimate the most likely values for those parameters.

We will study several effects that could modify the reference cross sections. We parametrize these effects in terms of modifications of the $\mathrm{CC}$ and $\mathrm{NC}$ cross sections and their respective inelasticities. Each particular case would give an expression for $N_{x, k}^{\mathcal{Z}}(\boldsymbol{\theta})$ in terms of the reference values (10) and the parameters $\boldsymbol{\theta}$. Putting these expressions in (13) will give us the most likely parameters and the confidence contours in the parameter space. Table II 
TABLE II. Observed/expected number of events in each category.

\begin{tabular}{lcc}
\hline \hline Event direction & Shower & Track \\
\hline Downgoing & $18 / 19.8$ & $6 / 4.2$ \\
Upgoing & $5 / 11.5$ & $7 / 2.5$ \\
\hline \hline
\end{tabular}

contains the expected number of events in each one of the four categories compared to the observed ones.

\section{A. Probing strong dynamics with IceCube data}

The kinematics of lepton-nucleon scattering is described in terms of the variables $Q^{2}$, Bjorken $x$, and the inelasticity $y=Q^{2} / s x$ that measures the energy transfer between the lepton and nucleon systems, with $s=2 E_{\nu} m_{N}$ the square of the center-of-mass energy. The cross section for $\mathrm{CC}$ neutrino (and antineutrino) scattering on isoscalar nucleon targets is given by [36]

$$
\sigma_{\mathrm{CC}, 0}=\int_{0}^{1} d x \int_{0}^{x s} d Q^{2} \frac{d^{2} \sigma^{\nu(\bar{\nu}) N}}{d x d Q^{2}}
$$

where

$$
\begin{aligned}
\frac{d^{2} \sigma^{\nu(\bar{\nu}) N}}{d x d Q^{2}}= & \frac{G_{\mathrm{F}}^{2}}{2 \pi x}\left(\frac{m_{W}^{2}}{Q^{2}+m_{W}^{2}}\right)^{2}\left[Y_{+} F_{2}^{\nu(\bar{\nu})}\left(x, Q^{2}\right)\right. \\
& \left.-y F_{\mathrm{L}}^{\nu(\bar{\nu})}\left(x, Q^{2}\right)+Y_{-} x F_{3}^{\nu(\nu)}\left(x, Q^{2}\right)\right]
\end{aligned}
$$

is the differential cross section given in terms of the structure functions $F_{2}^{\nu(\bar{\nu})}, F_{\mathrm{L}}^{\nu(\bar{\nu})}$, and $x F_{3}^{\nu(\bar{\nu})}$, and $Y_{+}=1+(1-y)^{2}, Y_{-}=1-(1-y)^{2}$. Here, $G_{\mathrm{F}}$ is the Fermi constant and $m_{W}$ is the $W$-boson mass. At leading order (LO) in perturbative QCD, the structure functions are given in terms of parton distributions as $F_{2}^{\nu(\bar{\nu})}=x\left[\sum_{i} \alpha_{i} q_{i}\left(x, Q^{2}\right)+\sum_{j} \alpha_{j} \bar{q}_{j}\left(x, Q^{2}\right)\right], \quad x F_{3}^{\nu(\bar{\nu})}=$ $x\left[\sum_{i} \beta_{i} q_{i}\left(x, Q^{2}\right)+\sum_{j} \beta_{j} \bar{q}_{j}\left(x, Q^{2}\right)\right]$ and $F_{\mathrm{L}}^{\nu(\bar{\nu})}=0$ [36]. For neutrinos, $i=u, d, s, b$ and $j=u, d, c$, with $\alpha_{i}=$ $\alpha_{j}=\beta_{i}=1$ for $u, d ; \alpha_{i}=\alpha_{j}=\beta_{i}=2$ for $s, b ; \beta_{j}=-1$ for $u, d ; \beta_{j}=-2$ for $c$ quarks. For antineutrinos, $i=u, d, c$ and $j=u, d, s, b$, with $\alpha_{i}=\alpha_{j}=\beta_{i}=1$ for $u, d$; $\alpha_{i}=$ $\alpha_{j}=\beta_{i}=2$ for $c ; \beta_{j}=-1$ for $u, d ; \beta_{j}=-2$ for $s, b$ quarks.

The NC cross sections on isoscalar targets are given by expressions similar to (14) and (15), with the $W$ propagator replaced by the $Z$ propagator. For NC interactions the LO expressions for the structure functions are given by $F_{2}^{\nu(\bar{\nu})}=x\left\{\sum_{i} \alpha_{i}\left[q_{i}\left(x, Q^{2}\right)+\bar{q}_{i}\left(x, Q^{2}\right)\right]+\sum_{j} \alpha_{j}\left[q_{j}\left(x, Q^{2}\right)+\right.\right.$ $\left.\left.\bar{q}_{j}\left(x, Q^{2}\right)\right]+\sum_{k} \alpha_{k}\left[q_{k}\left(x, Q^{2}\right)+\bar{q}_{k}\left(x, Q^{2}\right)\right]\right\}$ and $x F_{3}^{\nu(\bar{\nu})}=$ $\sum_{i} x\left(v_{u} a_{u}+v_{d} a_{d}\right)\left[q_{i}\left(x, Q^{2}\right)-\bar{q}_{i}\left(x, Q^{2}\right)\right]$, where $i=u$, $d, \quad j=s, \quad b, \quad k=c, \quad \alpha_{i}=\left(a_{u}^{2}+v_{u}^{2}+a_{d}^{2}+v_{d}^{2}\right) / 2$, $\alpha_{j}=a_{d}^{2}+v_{d}^{2}$, and $\alpha_{k}=a_{u}^{2}+v_{u}^{2}$, with $v_{u}, v_{d}, a_{u}, a_{d}$ the
NC vector and axial couplings for $u$ - and $d$-type quarks [36].

At next-to-leading order (NLO) the $F$-functional relations involve further QCD-calculable coefficient functions and contributions from $F_{\mathrm{L}}$ can no longer be neglected [23]. The parton distribution functions (PDFs) are determined in fits to deep inelastic scattering (DIS) data by the following procedure. The PDFs are parametrized at some initial scale $Q_{0} \sim 1 \mathrm{GeV}$ and then evolved, using the NLO DGLAP equations [37-40], to higher values of $Q^{2}$. They are then convoluted with QCD-calculable coefficient functions to give NLO predictions for the structure functions, which are then fitted to the DIS data, to obtain the CC and $\mathrm{NC}$ neutrino-nucleon cross sections shown in Fig. 1 [15].

To probe the PDFs, we assume a simple global scaling of the total reference cross section, $\sigma_{\text {tot }}=S \sigma_{\text {tot, }, 0}$, and thus $\alpha_{i}=\alpha_{i, 0}$. We further assume the inelasticity of the $\mathrm{NC}$ interaction remains unchanged, and so $a_{\alpha_{0}}=a_{\alpha}$. With this in mind, the set of parameters for the likelihood analysis is $\boldsymbol{\theta}=\{F, S\}$, and the expressions in (5) become

$$
N_{d}^{\mathcal{Z}}=F S \sum_{(i, \alpha) \in \mathcal{Z}} \tilde{N}_{d}^{i, \alpha}
$$

$$
N_{u}^{\mathcal{Z}}=F \sum_{(i, \alpha) \in \mathcal{Z}} \tilde{N}_{u}^{i, \alpha}
$$

for $\mathcal{Z} \in\{\mathcal{S}, \mathcal{T}\}$.

The likelihood maximizes for the pair of values

$$
\left\{\begin{array}{l}
S=1.16_{-0.34}^{+0.51}(1 \sigma \text { C.L. }), \\
F=0.86_{-0.22}^{+0.27}(1 \sigma \text { C.L. }) .
\end{array}\right.
$$

In Fig. 3 we show the confidence contours and the associated curves in the $F-S$ plane for each event type that would produce the observed number of events of each type. In Fig. 4 we show the profile likelihood ratio for $S$. Note that the cross section is consistent at the $1 \sigma$ level with the value obtain from perturbative QCD calculations guided by HERA data, and IceCube measurement [14,17]. However, thus far the study is statistics limited, with about $37 \%$ uncertainty. Note that because we have combined various energy bins there is a dependence of the cross section with the flux normalization, but is an almost negligible; see Appendix B for details. Of course, in a more general analysis considering an anisotropic flux of neutrinos and flavor ratios not equally distributed on Earth, additional free parameters need to be added to the likelihood analysis to account for the extra degrees of freedom (d.o.f.).

IceCube is also opening other doors to look for heavy new physics. Even if the mean inelasticity measured by IceCube up to $10^{6} \mathrm{GeV}$ is in agreement with the SM prediction [41], the energy dependence of the neutrinonucleon cross section [17] seems to leave some room for 


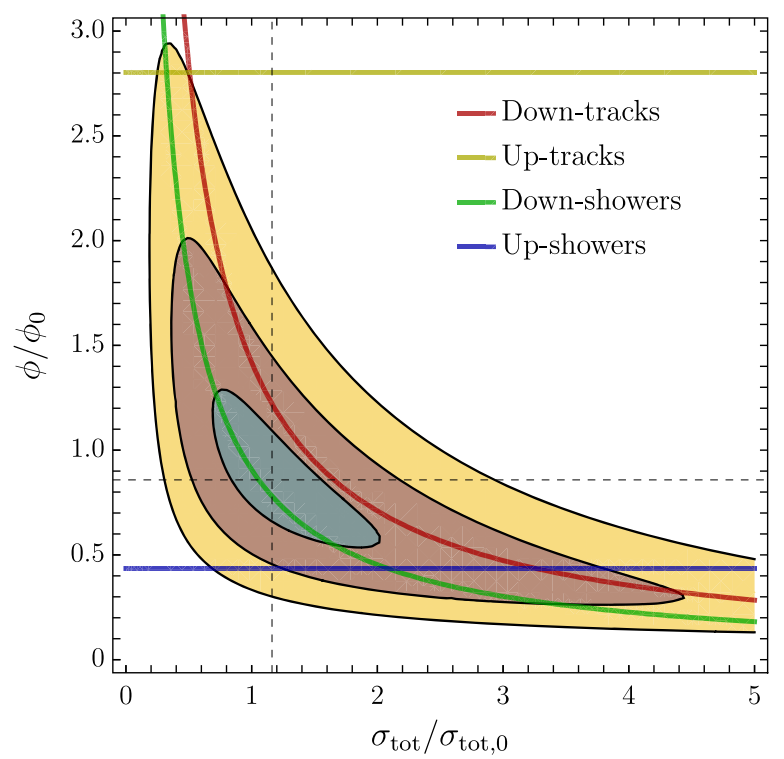

FIG. 3. 1, 3, and $5 \sigma$ confidence contours for $(F, S)$ for scaled total cross section $\sigma_{\text {tot }}$ and flux $\phi$ with respect to their reference values $\sigma_{\text {tot }, 0}, \phi_{0}$.

small new physics contributions affecting neutrino interactions both in [42] and beyond [43-45] the SM. Note that all of these processes would only increase the NC contribution to the neutrino-nucleon cross section, thus modifying the (perturbative) SM prediction of the $\sigma_{\mathrm{CC}} / \sigma_{\mathrm{NC}}$ ratio.

Next, in line with our stated plan, we duplicate our analysis but keeping the ratio $\sigma_{\mathrm{CC}} / \sigma_{\mathrm{NC}}$ as a free parameter in the likelihood function. The dichotomy between tracks (which are only produced via $\mathrm{CC}$ interactions) and showers provides a direct test of the $\sigma_{\mathrm{CC}} / \sigma_{\mathrm{NC}}$ ratio. Before proceeding we note that in the analysis carried out by the IceCube Collaboration [14] only upward going tracks are considered to keep the angular distribution of events with small uncertainties. Because of this, their analysis sets a limit

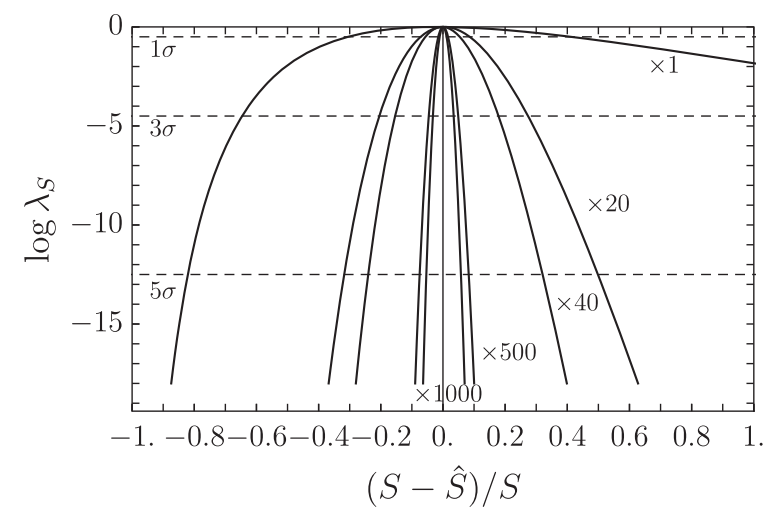

FIG. 4. Profile likelihood ratio for $S$, of the IceCube data analyzed in Fig. 3, and the four simulated samples seen in Figs. 6 and 8. Since different samples provide different estimates for $S$, the horizontal axis is rescaled to show all curves peaking at the same point, favoring visualization. on the charged-current neutrino nucleon cross section $\sigma_{\mathrm{CC}}$. In the analysis of [17] only the shower-HESE data sample is considered, with full scrutiny of the angular distribution of IceCube events. However, the ratio of the CC and NC contributions is fixed to that expected in the perturbative SM, i.e., $\sigma_{\mathrm{CC}} / \sigma_{\mathrm{NC}}=3$.

We begin by writing the total neutrino-nucleon cross section as $\sigma_{\mathrm{tot}}=\sigma_{\mathrm{CC}, 0}+\sigma_{\mathrm{NC}}$. Instead of considering the full scaling of the cross section $S$ as the parameter of interest, we set out the analysis to constrain the ratio $S_{\mathrm{NC}} \equiv \sigma_{\mathrm{NC}} / \sigma_{\mathrm{NC}, 0}$. Following a process similar to that used to obtain (5) from (3b), the expected numbers of downgoing events are

$$
\begin{aligned}
N_{d}^{\mathcal{S}} & =F\left(\sum_{\alpha=e, \tau} \tilde{N}_{d}^{\mathrm{CC}, \alpha}+S_{\mathrm{NC}} \sum_{\alpha=e, \mu, \tau} \tilde{N}_{d}^{\mathrm{NC}, \alpha}\right), \\
N_{d}^{\mathcal{T}} & =F \tilde{N}_{d}^{\mathrm{CC}, \mu} .
\end{aligned}
$$

Likewise, for upgoing events,

$$
\begin{aligned}
& N_{u}^{\mathcal{S}}=F\left(\sum_{\alpha=e, \tau} f_{\alpha}\left(S_{\mathrm{NC}}\right) \tilde{N}_{u}^{\mathrm{CC}, \alpha}+S_{\mathrm{NC}} \sum_{\alpha=e, \mu, \tau} f_{\alpha}\left(S_{\mathrm{NC}}\right) \tilde{N}_{u}^{\mathrm{N} C, \alpha}\right) \\
& N_{u}^{\mathcal{T}}=F f_{\mu}\left(S_{\mathrm{NC}}\right) \tilde{N}_{u}^{\mathrm{CC}, \mu}
\end{aligned}
$$

where

$$
\begin{gathered}
f_{\alpha}\left(S_{\mathrm{NC}}\right) \equiv \frac{1+r_{\alpha}}{Y_{\mathrm{NC}} S_{\mathrm{NC}}+r_{\alpha}}, \\
r_{\alpha} \equiv \frac{y_{\mathrm{CC}, 0}^{\alpha}}{y_{\mathrm{NC}, 0}^{\alpha}} \frac{\sigma_{\mathrm{CC}, 0}}{\sigma_{\mathrm{NC}, 0}},
\end{gathered}
$$

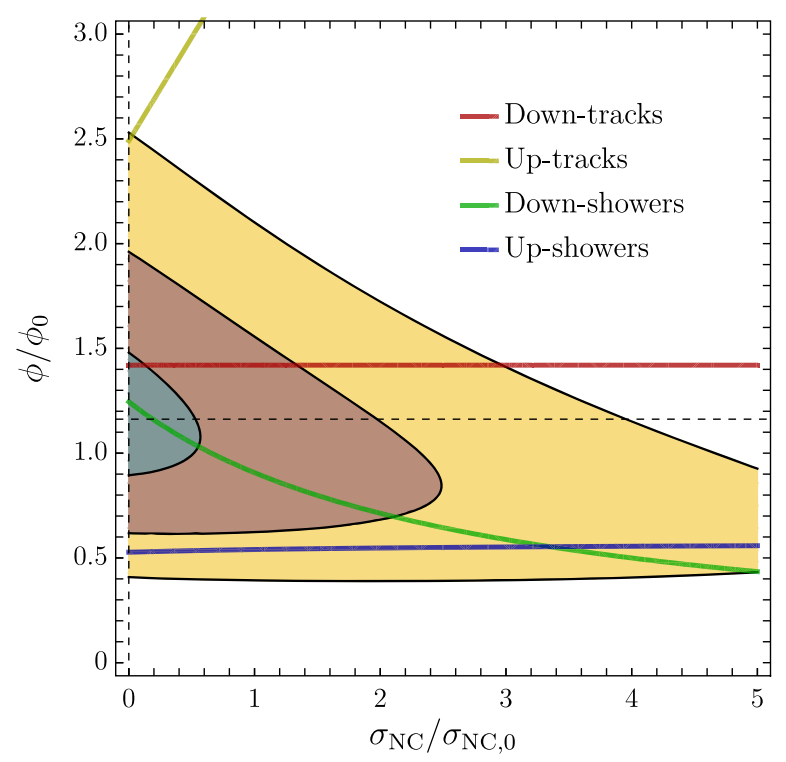

FIG. 5. 1, 3, and $5 \sigma$ confidence contours in the $\left(F, S_{\mathrm{NC}}\right)$ plane. 

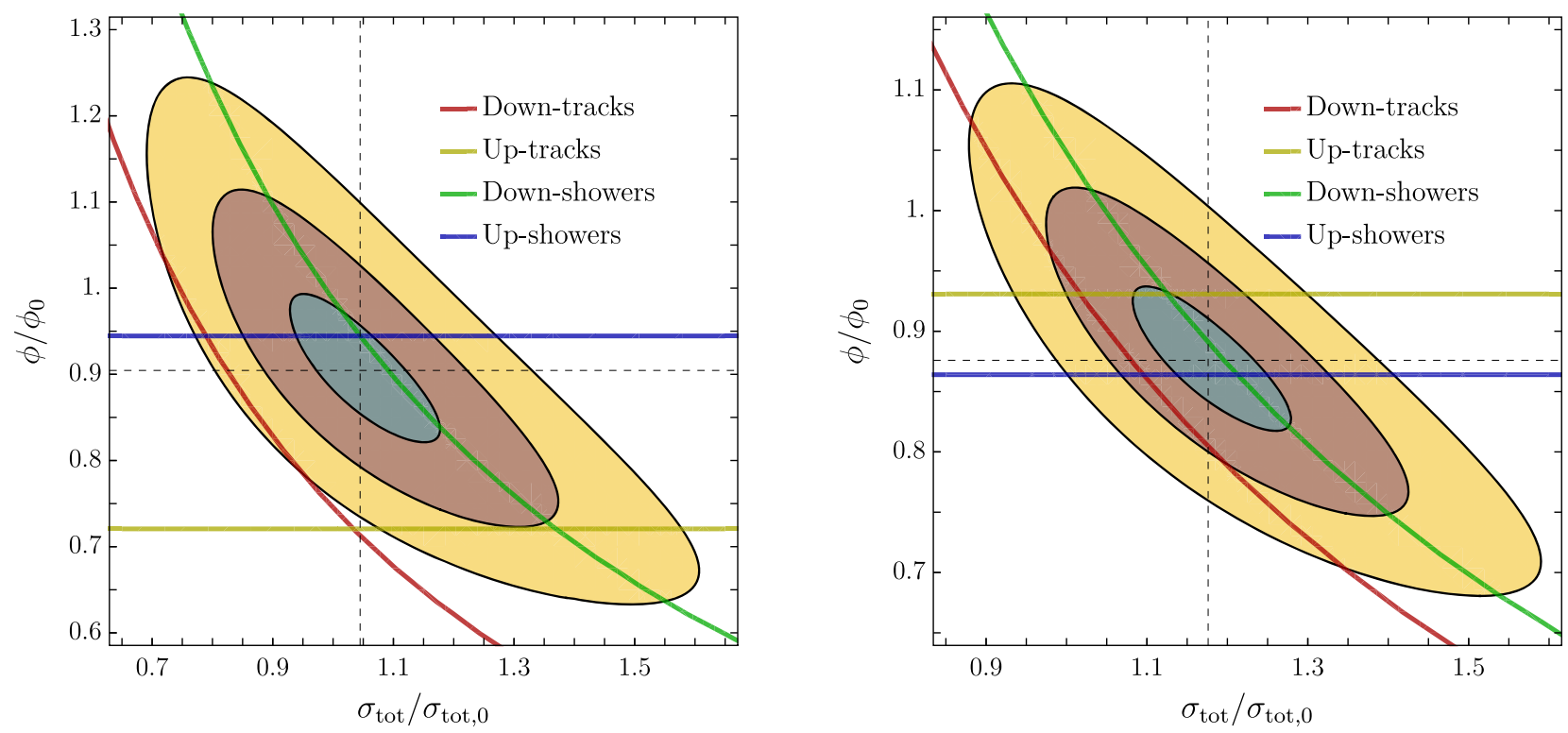

FIG. 6. Projected determinations of neutrino fluxes and cross sections at $\sqrt{s} \sim 1$ TeV from future IceCube-Gen 2 data. The 1,3 , and $5 \sigma$ confidence contours are based on simulated data for a $20 \times$ (left) and $40 \times$ (right) the actual IceCube sample.

and $Y_{\mathrm{NC}}^{\alpha} \equiv y_{\mathrm{NC}}^{\alpha} / y_{\mathrm{NC}, 0}^{\alpha}$, and where we have assumed that the average inelasticities for $\mathrm{CC}$ interactions remain unchanged from those of the SM. For the SM values, and assuming flavor independent inelasticities, we can approximate $r_{\alpha} \approx 8 \equiv r$. In such case, the upgoing expected event numbers are simplified to

$N_{u}^{\mathcal{S}}=F \frac{1+r}{Y_{\mathrm{NC}} S_{\mathrm{NC}}+r}\left(\sum_{\alpha=e, \tau} \tilde{N}_{u}^{\mathrm{CC}, \alpha}+S_{\mathrm{NC}} \sum_{\alpha=e, \mu, \tau} \tilde{N}_{u}^{\mathrm{NC}, \alpha}\right)$

$N_{u}^{\mathcal{T}}=F \frac{1+r}{Y_{\mathrm{NC}} S_{\mathrm{NC}}+r} \tilde{N}_{d}^{\mathrm{CC}, \mu}$.

Data from the Large Hadron Collider (LHC) put severe constraints on stringy and gravity contributions to the neutrino-nucleon scattering cross section [46-50]. However, nonperturbative SM processes, such as sphaleron transitions, remain almost unconstrained by LHC data [50]. By comparing the 90 fermionic d.o.f. in the SM with the 6 d.o.f. in the neutrino sector contributing to missing energy $\boldsymbol{E}_{T}$, we take $y_{\mathrm{NC}} \simeq 0.95$ and so using $y_{\mathrm{NC}, 0} \simeq 0.3$ we have $Y_{\mathrm{NC}} \simeq 3$. This particular choice of $y_{\mathrm{NC}}$ is also valid for excitations of the string and quantum black hole production in scenarios with large extra-dimensions. ${ }^{2}$ However, this is not the case for exchange of Kaluza-

\footnotetext{
${ }^{2}$ To first approximation, the black hole can be treated as a point-radiator that emits mostly $s$-waves. This indicates that it decays equally to a particle on the brane and in the bulk, since it is only sensitive to the radial coordinate and does not make use of the extra angular modes available in the bulk. Since there are many more particles on our brane than in the bulk, this has the crucial consequence that the black hole decays visibly to SM particles [51,52].
}

Klein gravitons in the large extra-dimension braneworld, where the transferred energy fraction is only around $0.1[53,54]$.

Maximizing the likelihood (13) for the parameters $\boldsymbol{\theta}=\left\{F, S_{\mathrm{NC}}\right\}$ using (18) and (22) provides the values

$$
\left\{\begin{aligned}
S_{\mathrm{NC}} & =0.00_{-0.00}^{+0.27}(1 \sigma \text { C.L. }), \\
F & =1.16_{-0.18}^{+0.20}(1 \sigma \text { C.L. }) .
\end{aligned}\right.
$$

In Fig. 5 we show the confidence contours and the associated curves in the $F-S_{\mathrm{NC}}$ plane for each event type that would produce the observed number of events of each type. We can conclude that $S_{\mathrm{NC}}>1$ is excluded at $2 \sigma$ level.

In summary, we have used the complete $(\mathcal{S}+\mathcal{T})$ HESE data sample to constrain the rise of $\sigma_{\mathrm{NC}}$. Because the data are scant and the arrival direction of shower events have large uncertainties we have chosen to integrate over the angular distribution. Note that the analysis presented herein is complementary to those reported in $[14,17]$ as it test a different region of the neutrino-nucleon cross section parameter space. Indeed, the likelihood fit given in (23) provides the first unequivocal constraint derived from IceCube data on nonperturbative SM phenomena [42], low-mass-string-scale Regge excitations [43], and gravity effects [2].

\section{B. Looking ahead with IceCube-Gen2}

Design studies for the IceCube-Gen2 high-energy array are well underway [18]. They will result in an instrumented volume approaching $10 \mathrm{~km}^{3}$ and will lead to significantly larger neutrino detection rates, across all neutrino flavor and detection channels. A rough estimate indicates about an 


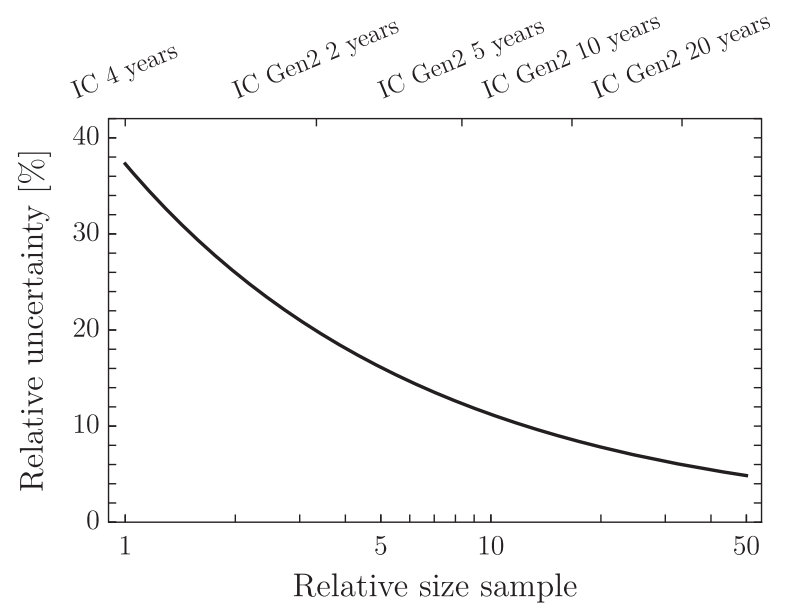

FIG. 7. Evolution of the cross section precision measurement.

order of magnitude increase in exposure per year. The bigger instrumented volume will facilitate the calorimetric detection of muon tracks, reducing significantly the systematic uncertainty. The extension will reuse the very reliable design of IceCube's digital optical modules and therefore it will surely perform technologically at least at the level of IceCube. A conservative estimate of the sample size is then attainable by simply scaling the instrumented volume.

To determine the sensitivity of IceCube-Gen2 to probe strong dynamics, we generate random samples of events, $\bar{N}_{x, k}^{\mathcal{Z}}$, following the distribution (11), with the parameters for a scaled total cross section found in the IceCube data analysis, which are summarized in (17). To accommodate the bigger instrumented volume we adopt a multiplying factor on the IceCube data sample. In 10 years of observation IceCube-Gen2 will collect about 500 neutrino

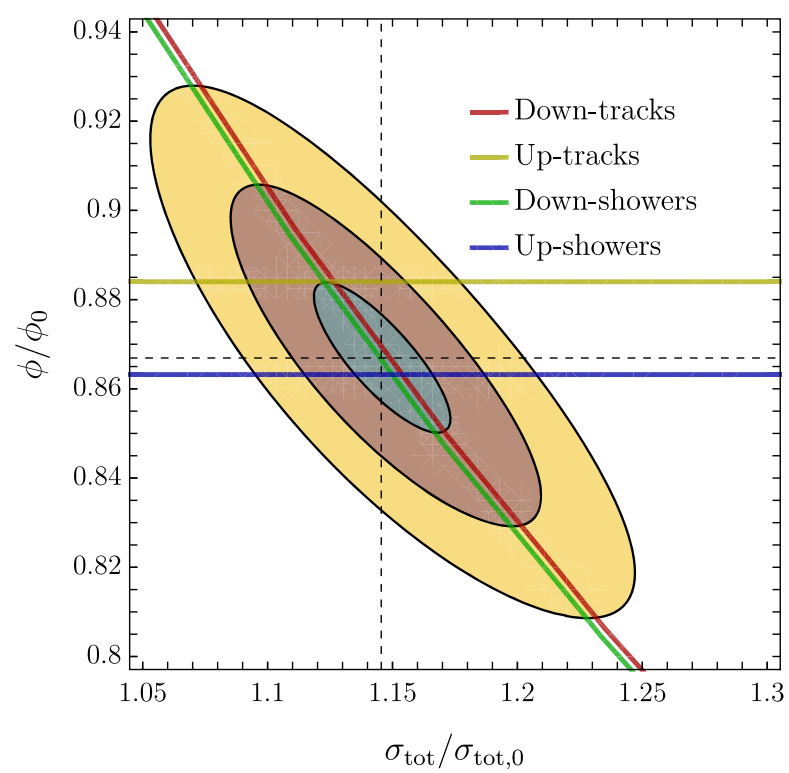

events in the energy range $0.1 \lesssim E_{\nu} / \mathrm{PeV} \lesssim 2$, and about 1000 events in 20 years. Thus we adopt 20 and 40 as the representative multiplicative factors associated with these data samples. Using the high-energy and high-statistics sample to be collected by IceCube-Gen2, we perform the same likelihood analysis as with the real data. The results are shown in Fig. 6 for a sample of $20 \times$ and $40 \times$ the actual sample size. The precision on the cross section determination would be $7.9 \%$ and $5.5 \%$ for $\sim 500$ and $\sim 1000$ events, respectively. This precision is comparable to that obtained in perturbative QCD calculations guided by HERA data. Of course this will also require a comparable reduction on the systematic uncertainties, otherwise any study would become systematics-limited. Detailed evolution of the uncertainty with sample sizes is illustrated in Fig. 7.

We can also envisage an IceCube-like detector of $100 \mathrm{~km}^{3}$, specifically designed to probe strong dynamics. In Fig. 8 we entertain this possibility and show the results of a likelihood analysis based on simulated data for a $500 \times$ and a $1000 \times$ the actual sample. The $1 \sigma$ contour regions could reach a precision of less than $2 \%$ level.

Some of the technologies needed to enable the next generation neutrino observatories are already in development. For example, the strings of IceCube-Gen2 will use multi-PMT DOMs, providing better directionality and more than double the photocathode area per module [55-57]. The new instrumentation will dramatically boost IceCube-Gen2 performance. The strings will feature new calibration devices that would allow us to better model the optical properties of the ice, reducing systematic uncertainties in the tau neutrino appearance measurement, and improving reconstruction of the direction of high energy cascade events. The reduction of systematics uncertainties

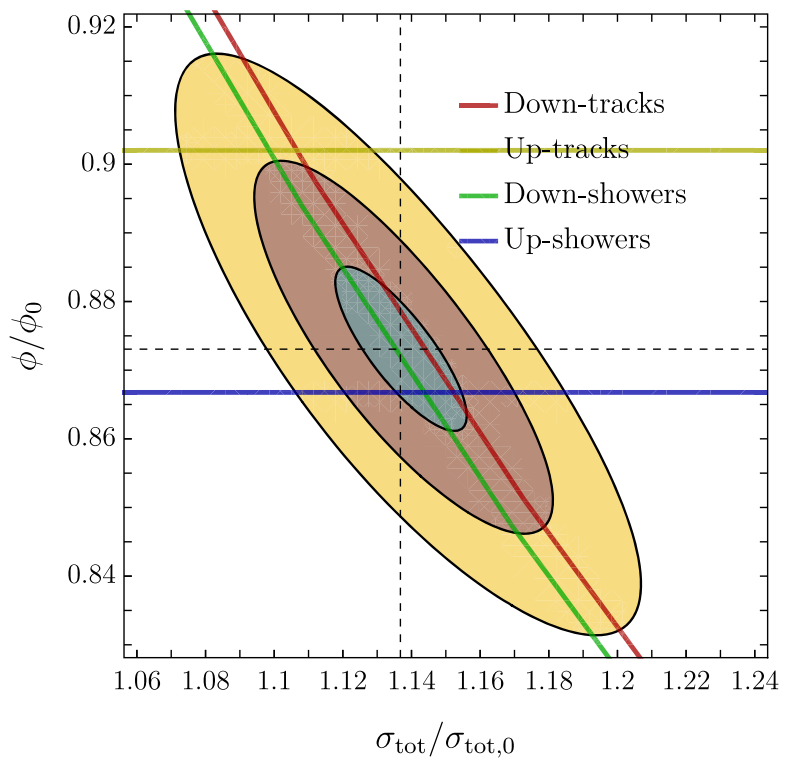

FIG. 8. Confidence contours based in simulated data for a $500 \times($ left) and $1000 \times$ (right) the actual sample. 
in the arrival direction of $\mathcal{S}$-events would play a pivotal role in the accurate determination of the neutrino-nucleon cross section. Strategies and new devices to greatly improve the angular resolution of next-to-next-generation cosmic neutrino detectors are also under discussion. Any detailed discussion addressing the challenges to be faced in the design of these facilities falls outside the scope of this article, which has been planned as a phenomenological approach to neutrino scattering on ice.

\section{CONCLUSIONS}

Motivated by IceCube observations we have reexamined a technique to probe strong dynamics with neutrino telescopes in the Antarctic ice. The strategy involves comparing the rate for upgoing and downgoing neutrino events to disentangle effects from the unknown flux and those from QCD dynamics. More concretely, we implemented the standard statistical analysis, using two uncorrelated observables (up- and down-going events), to determine the best fit model parameters (flux and cross section) and the fluctuations around the favored values. The hypotheses of the model being tested are: (i) an isotropic neutrino flux and (ii) flavor ratios democratically distributed on Earth, both consistent with IceCube data [30-33,35]. Current results from IceCube already provide interesting constraints on the flux cross-section parameter space. Using $6 \mathrm{yr}$ of IceCube HESE data we have obtained a measurement of the neutrino-nucleon cross section at $\sqrt{s} \sim 1 \mathrm{TeV}$. We have shown that the measured cross section is consistent within $1 \sigma$ with perturbative QCD calculations constrained by HERA measurements, and also with the recent IceCube measurement $[14,17]$. Note that with current statistics in the HESE data-sample our measurement has a $37 \%$ uncertainty, compared to the measured cross section with $35 \%$ uncertainty reported by the IceCube Collaboration using a larger data sample, and the 5\% error of the SM experimentally constrained calculation using HERA data. In a separate study we have also constrained contributions from nonperturbative processes to the neutrino-nucleon cross section. We have shown that contributions to the NC interaction at $\sqrt{s} \sim 1 \mathrm{TeV}$ from electroweak sphaleron transitions are excluded at the $2 \sigma$ level.

However, the most important result of our study is the investigation on the potential of future neutrino-detection facilities for measuring the neutrino-nucleon cross section. Using the energy and angular distributions observed by the IceCube neutrino detector, we have demonstrated that in the near future IceCube-Gen2 will carry striking improvements to determine both astrophysical neutrino fluxes and cross section. In particular, we have shown that the highenergy high-statistics data sample to be collected by this facility will fetch a direct measurement of the neutrinonucleon cross section at $\sqrt{s} \sim 1 \mathrm{TeV}$, with a precision of about a $6 \%$, that is comparable to perturbative QCD informed by HERA data. We have also shown that a
$100 \mathrm{~km}^{3}$ detector would reach the unprecedented precision of less than a $2 \%$ level.

\section{ACKNOWLEDGMENTS}

We thank Subir Sarkar for permission to reproduce Fig. 1. L. A. A. and J.F.S. are supported by U.S. National Science Foundation (NSF Grant No. PHY1620661) and by the National Aeronautics and Space Administration (NASA 80NSSC18K0464). C. G. C. is supported by ANPCyT. Any opinions, findings, and conclusions or recommendations expressed in this material are those of the authors and do not necessarily reflect the views of NSF or NASA.

\section{APPENDIX A: UPGOING EVENT RATE}

The probability for a neutrino with incident angle $\vartheta$ measured from the horizon and azimuth angle $\varphi$ to survive for a distance $x$ along a chord through the Earth is given by

$$
P_{\text {survival }}(x)=e^{-x / \lambda_{a}},
$$

where $\lambda_{a}=\left(\sigma_{a} \rho_{\oplus} N_{A}\right)^{-1}$ is the neutrino attenuation length, with $N_{A}=6.022 \times 10^{23} \mathrm{~g}^{-1}$ and $\rho_{\oplus}$ the Earth's density, and where $\sigma_{a}=\sum_{i} \sigma_{i} y_{i}$ is the attenuation cross section, defined as the total cross section weighted by the inelasticity $y_{i}$, with $i \in\{\mathrm{CC}, \mathrm{NC}\}$ [58]. The probability for neutrino interaction producing an observable signal (either via a $\mathrm{CC}$ or a $\mathrm{NC}$ process) in the interval $(x, x+d x)$ is

$$
P_{\text {signal }}(x)=\frac{d x}{\lambda_{i}}
$$

where $\lambda_{i}=\left(\sigma_{i} \rho_{\oplus} N_{A}\right)^{-1}$ is the neutrino interaction length. The neutrino traverses a chord length $l=2 R_{\oplus} \sin \vartheta$, with $R_{\oplus}$ the Earth's radius. Note that $\lambda_{a}$ limits the maximum chord length, and therefore the solid angle over which neutrinos can be observed scales as $\Omega=2 \pi \sin \vartheta=$

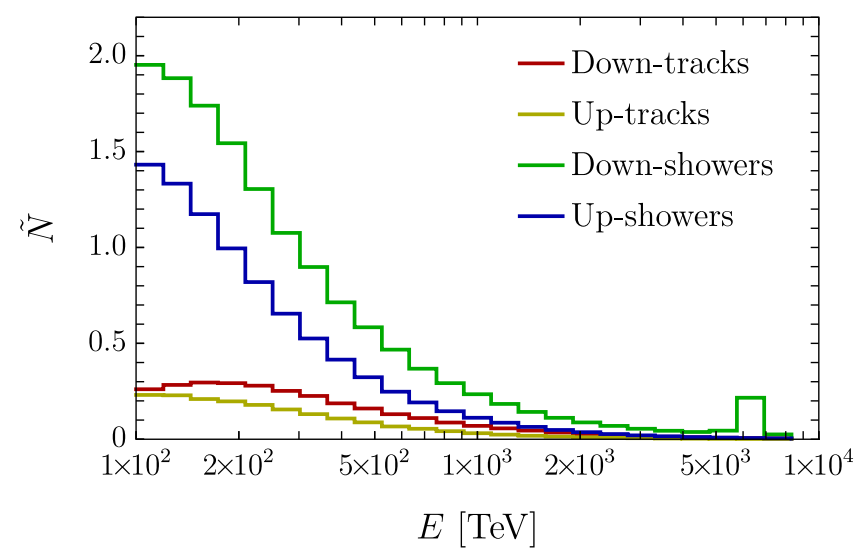

FIG. 9. Reference number of events from (10) with normalization given by (B1). 
TABLE III. Observed/expected number of events in each category.

\begin{tabular}{lcc}
\hline \hline Event direction & Shower & Track \\
\hline Down-going & $18 / 14.1$ & $6 / 2.9$ \\
Up-going & $5 / 8.7$ & $7 / 1.8$ \\
\hline \hline
\end{tabular}

$\pi \lambda_{a} / R_{\oplus}$ [5]. For a given neutrino flux $\phi$, the rate of upgoing events at IceCube is then estimated to be

$$
\Gamma_{\text {up }}=\phi A_{\nu} \frac{d \varphi}{d \Omega} \int_{\ell}^{2 R_{\oplus}} 2 \pi \frac{d l}{2 R_{\oplus}} \int_{l-\ell}^{l} e^{-x / \lambda_{a}} \frac{d x}{\lambda_{i}},
$$

which on integration yields

$$
\Gamma_{\text {up }}=\phi \pi A_{\nu} \frac{d \varphi}{d \Omega} \frac{\lambda_{a}^{2}}{R_{\oplus} \lambda_{i}}\left(1-e^{-\ell / \lambda_{a}}\right)\left(1-e^{-\left(2 R_{\oplus}-\ell\right) / \lambda_{a}}\right),
$$

where $A_{\nu}$ is the area of the detector projected against the neutrino direction and $\ell$ is the portion of the neutrino path to which the detector is sensitive [1]. Note that the effective volume over which an interaction may be detected is $V_{\text {eff }}=A_{\nu} \ell$, where $\ell$ depends on: (i) the lepton stopping $(d E / d x)$ or decay, (ii) the chord length to the surface, and (iii) the detector size. For $2 R_{\oplus} \gg \lambda_{a} \gg \ell$, (A4) simplifies to

$$
\Gamma_{\text {up }} \simeq \phi \pi A_{\nu} \frac{d \varphi}{d \Omega} \frac{\ell}{R_{\oplus}} \frac{\lambda_{a}}{\lambda_{i}} \propto \phi \frac{\sigma_{i}}{\sigma_{a}} .
$$

For completeness, we note that to calculate the event rate for a surface detector (e.g., the Pierre Auger Observatory), we must include an additional factor of $\sin \vartheta=l /\left(2 R_{\oplus}\right)$ in the $d l$ integral to project out the normal component of the lepton flux emerging from the Earth, and so the rate of Earth-skimming neutrinos scales as $\phi \sigma_{i} / \sigma_{a}^{2}$, as shown in [2].

\section{APPENDIX B: DEPENDENCE ON THE NEUTRINO SPECTRUM}

As we have pointed out Sec. II, for a given bin of energy, we can constrain neutrino interactions without assuming particular neutrino fluxes or cross sections. However, because of the limited data sample we have combined the results of various energy bins. This introduces a dependence of the neutrino-nucleon cross section with the shape of the neutrino spectrum.

To estimate the uncertainty associated with the spectral shape, in what follows we duplicate the procedure of

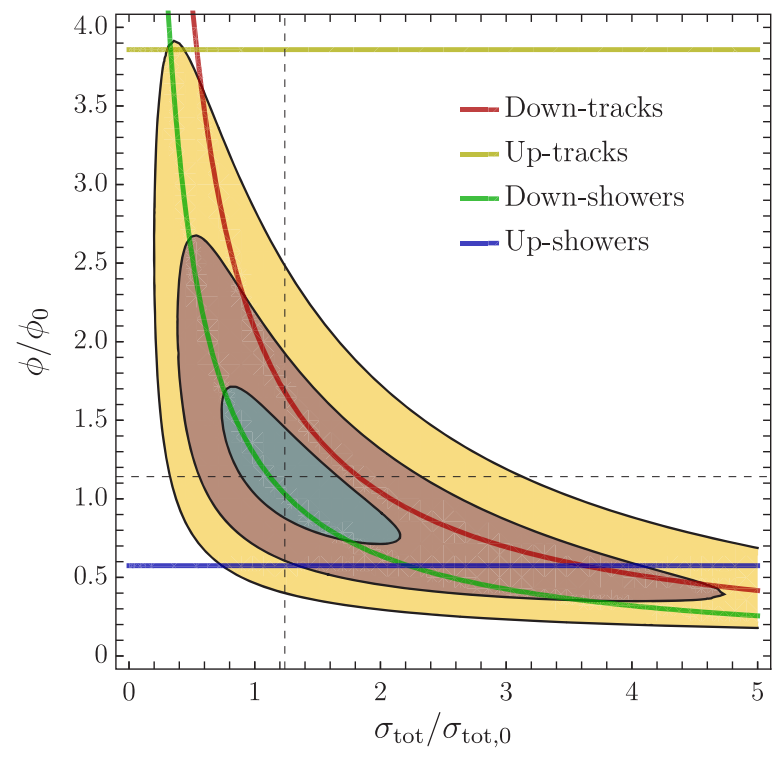

FIG. 10. 1, 3, and $5 \sigma$ confidence contours for $(F, S)$ for scaled total cross section $\sigma_{\text {tot }}$ and flux $\phi$ with respect to their reference values $\sigma_{\text {tot }, 0}$ and $\phi_{0}$ as given by (B1).

Sec. III A, but with a flux normalization given by the most recent fit to the spectrum by the IceCube Collaboration,

$\phi_{0}=N_{\phi}\left(\frac{E_{\nu}}{100 \mathrm{TeV}}\right)^{-\gamma} \times 10^{-18}\left(\mathrm{GeV} \mathrm{cm}^{2} \mathrm{~s} \mathrm{sr}\right)^{-1}$,

where $N_{\phi}=6.45_{-0.46}^{+1.46}$ and $\gamma=2.89_{-0.19}^{+0.20}$ [59]. The values of the expected number of events considering the central values of the flux given in (B1) are shown in Fig. 9. Table III contains the expected number of events in each one of the four categories compared to the observed ones.

For the ratios given in Table III, the likelihood maximizes for the pair of values

$$
\left\{\begin{array}{l}
S=1.24_{-0.36}^{+0.54}(1 \sigma \text { C.L. }) \\
F=1.14_{-0.30}^{+0.36}(1 \sigma \text { C.L. })
\end{array}\right.
$$

In Fig. 10 we show the confidence contours and the associated curves in the $F-S$ plane for each event type that would produce the observed number of events of each type.

Note that by considering the steeper spectrum given in (B1) the error in the cross section slightly improves from $37 \%$ to $36 \%$. We conclude that the determination of the neutrino-nucleon cross section carried out in Sec. III A is robust. 
[1] A. Kusenko and T. J. Weiler, Neutrino Cross-Sections at High-Energies and the Future Observations of UltrahighEnergy Cosmic Rays, Phys. Rev. Lett. 88, 161101 (2002).

[2] L. A. Anchordoqui, J. L. Feng, H. Goldberg, and A. D. Shapere, Black holes from cosmic rays: Probes of extra dimensions and new limits on TeV scale gravity, Phys. Rev. D 65, 124027 (2002).

[3] L. A. Anchordoqui, J. L. Feng, and H. Goldberg, Particle Physics on Ice: Constraints on Neutrino Interactions far above the Weak Scale, Phys. Rev. Lett. 96, 021101 (2006).

[4] L. A. Anchordoqui, A. M. Cooper-Sarkar, D. Hooper, and S. Sarkar, Probing low- $x$ QCD with cosmic neutrinos at the Pierre Auger Observatory, Phys. Rev. D 74, 043008 (2006).

[5] S. Hussain, D. Marfatia, D. W. McKay, and D. Seckel, Cross Section Dependence of Event Rates at Neutrino Telescopes, Phys. Rev. Lett. 97, 161101 (2006).

[6] E. Borriello, A. Cuoco, G. Mangano, G. Miele, S. Pastor, O. Pisanti, and P.D. Serpico, Disentangling neutrinonucleon cross section and high energy neutrino flux with a $\mathrm{km}^{3}$ neutrino telescope, Phys. Rev. D 77, 045019 (2008).

[7] L. A. Anchordoqui, Ultra-high-energy cosmic rays, Phys. Rep. 801, 1 (2019).

[8] M. G. Aartsen et al. (IceCube Collaboration), First Observation of PeV-Energy Neutrinos with IceCube, Phys. Rev. Lett. 111, 021103 (2013).

[9] M. G. Aartsen et al. (IceCube Collaboration), Evidence for high-energy extraterrestrial neutrinos at the IceCube detector, Science 342, 1242856 (2013).

[10] M. G. Aartsen et al. (IceCube Collaboration), Observation of High-Energy Astrophysical Neutrinos in Three Years of IceCube Data, Phys. Rev. Lett. 113, 101101 (2014).

[11] M. G. Aartsen et al. (IceCube Collaboration), Atmospheric and astrophysical neutrinos above $1 \mathrm{TeV}$ interacting in IceCube, Phys. Rev. D 91, 022001 (2015).

[12] M. G. Aartsen et al. (IceCube Collaboration), The IceCube neutrino observatory-Contributions to ICRC 2015 Part II: Atmospheric and astrophysical diffuse neutrino searches of all flavors, arXiv:1510.05223.

[13] M. G. Aartsen et al. (IceCube Collaboration), The IceCube neutrino observatory contributions to ICRC 2017 Part II: Properties of the atmospheric and astrophysical neutrino flux, arXiv:1710.01191.

[14] M. G. Aartsen et al. (IceCube Collaboration), Measurement of the multi-TeV neutrino cross section with IceCube using Earth absorption, Nature (London) 551, 596 (2017).

[15] A. Cooper-Sarkar and S. Sarkar, Predictions for high energy neutrino cross-sections from the ZEUS global PDF fits, J. High Energy Phys. 01 (2008) 075.

[16] A. Cooper-Sarkar, P. Mertsch, and S. Sarkar, The high energy neutrino cross-section in the Standard Model and its uncertainty, J. High Energy Phys. 08 (2011) 042.

[17] M. Bustamante and A. Connolly, Extracting the EnergyDependent Neutrino-Nucleon Cross Section above $10 \mathrm{TeV}$ Using IceCube Showers, Phys. Rev. Lett. 122, 041101 (2019).

[18] M. G. Aartsen et al. (IceCube Collaboration), IceCubeGen2: A vision for the future of neutrino astronomy in Antarctica, arXiv:1412.5106.
[19] R. Gandhi, C. Quigg, M. H. Reno, and I. Sarcevic, Ultrahigh-energy neutrino interactions, Astropart. Phys. 5, 81 (1996).

[20] R. Gandhi, C. Quigg, M. H. Reno, and I. Sarcevic, Neutrino interactions at ultrahigh-energies, Phys. Rev. D 58, 093009 (1998).

[21] A. Connolly, R. S. Thorne, and D. Waters, Calculation of high energy neutrino-nucleon cross sections and uncertainties using the MSTW parton distribution functions and implications for future experiments, Phys. Rev. D 83, 113009 (2011).

[22] C. Y. Chen, P. S. Bhupal Dev, and A. Soni, Standard model explanation of the ultrahigh energy neutrino events at IceCube, Phys. Rev. D 89, 033012 (2014).

[23] C. A. Argelles, F. Halzen, L. Wille, M. Kroll, and M. H. Reno, High-energy behavior of photon, neutrino, and proton cross sections, Phys. Rev. D 92, 074040 (2015).

[24] V. Bertone, R. Gauld, and J. Rojo, Neutrino telescopes as QCD microscopes, J. High Energy Phys. 01 (2019) 217.

[25] R. Abbasi et al. (IceCube Collaboration), First search for atmospheric and extraterrestrial neutrino-induced cascades with the IceCube detector, Phys. Rev. D 84, 072001 (2011).

[26] L. A. Anchordoqui, M. M. Block, L. Durand, P. Ha, J. F. Soriano, and T.J. Weiler, Evidence for a break in the spectrum of astrophysical neutrinos, Phys. Rev. D 95, 083009 (2017).

[27] M. C. Gonzalez-Garcia and F. Halzen, Gamma ray burst neutrinos probing quantum gravity, J. Cosmol. Astropart. Phys. 02 (2007) 008.

[28] J. G. Learned and S. Pakvasa, Detecting tau-neutrino oscillations at PeV energies, Astropart. Phys. 3, 267 (1995).

[29] M. G. Aartsen et al. (IceCube Collaboration), Search for astrophysical tau neutrinos in three years of IceCube data, Phys. Rev. D 93, 022001 (2016).

[30] M. G. Aartsen et al. (IceCube Collaboration), Flavor Ratio of Astrophysical Neutrinos above $35 \mathrm{TeV}$ in IceCube, Phys. Rev. Lett. 114, 171102 (2015).

[31] O. Mena, S. Palomares-Ruiz, and A. C. Vincent, Flavor Composition of the High-Energy Neutrino Events in IceCube, Phys. Rev. Lett. 113, 091103 (2014).

[32] S. Palomares-Ruiz, A. C. Vincent, and O. Mena, Spectral analysis of the high-energy IceCube neutrinos, Phys. Rev. D 91, 103008 (2015).

[33] A. C. Vincent, S. Palomares-Ruiz, and O. Mena, Analysis of the 4-year IceCube high-energy starting events, Phys. Rev. D 94, 023009 (2016).

[34] D. Marfatia, D. W. McKay, and T. J. Weiler, New physics with ultra-high-energy neutrinos, Phys. Lett. B 748, 113 (2015).

[35] M. G. Aartsen et al. (IceCube Collaboration), Constraints on Galactic neutrino emission with seven years of IceCube data, Astrophys. J. 849, 67 (2017).

[36] R. Devenish and A. Cooper-Sarkar, Deep Inelastic Scattering (Oxford University Press, New York, 2004).

[37] G. Altarelli and G. Parisi, Asymptotic freedom in parton language, Nucl. Phys. B126, 298 (1977).

[38] V. N. Gribov and L. N. Lipatov, Deep inelastic ep scattering in perturbation theory, Yad. Fiz. 15, 781 (1972) [Sov. J. Nucl. Phys. 15, 438 (1972)]. 
[39] L. N. Lipatov, The parton model and perturbation theory, Yad. Fiz. 20, 181 (1974) [Sov. J. Nucl. Phys. 20, 94 (1975)].

[40] Y. L. Dokshitzer, Calculation of the structure functions for deep inelastic scattering and $e^{+} e^{-}$annihilation by perturbation theory in quantum chromodynamics, Zh. Eksp. Teor. Fiz. 73, 1216 (1977) [Sov. Phys. JETP 46, 641 (1977)].

[41] M. G. Aartsen et al. (IceCube Collaboration), Measurements using the inelasticity distribution of multi-TeV neutrino interactions in IceCube, Phys. Rev. D 99, 032004 (2019).

[42] J. Ellis, K. Sakurai, and M. Spannowsky, Search for sphalerons: IceCube vs. LHC, J. High Energy Phys. 05 (2016) 085.

[43] F. Cornet, J. I. Illana, and M. Masip, TeV Strings and the Neutrino Nucleon Cross-Section at Ultrahigh-Energies, Phys. Rev. Lett. 86, 4235 (2001).

[44] L. A. Anchordoqui, C. A. Garcia Canal, H. Goldberg, D. Gomez Dumm, and F. Halzen, Probing leptoquark production at IceCube, Phys. Rev. D 74, 125021 (2006).

[45] V. Barger and W. Y. Keung, Superheavy particle origin of IceCube PeV neutrino events, Phys. Lett. B 727, 190 (2013).

[46] G. Aad et al. (ATLAS Collaboration), Search for Quantum Black Hole Production in High-Invariant-Mass Lepton + Jet Final States Using $p p$ Collisions at $\sqrt{s}=8 \mathrm{TeV}$ and the ATLAS Detector, Phys. Rev. Lett. 112, 091804 (2014).

[47] A. M. Sirunyan et al. (CMS Collaboration), Search for narrow and broad dijet resonances in proton-proton collisions at $\sqrt{s}=13 \mathrm{TeV}$ and constraints on dark matter mediators and other new particles, J. High Energy Phys. 08 (2018) 130.

[48] M. Aaboud et al. (ATLAS Collaboration), Search for TeVscale gravity signatures in high-mass final states with leptons and jets with the ATLAS detector at $\sqrt{s}=13 \mathrm{TeV}$, Phys. Lett. B 760, 520 (2016).

[49] A. M. Sirunyan et al. (CMS Collaboration), Search for black holes in high-multiplicity final states in proton-proton collisions at $\sqrt{s}=13 \mathrm{TeV}$, Phys. Lett. B 774, 279 (2017).

[50] A. M. Sirunyan et al. (CMS Collaboration), Search for black holes and sphalerons in high-multiplicity final states in proton-proton collisions at $\sqrt{s}=13 \mathrm{TeV}$, J. High Energy Phys. 11 (2018) 042.

[51] R. Emparan, G. T. Horowitz, and R. C. Myers, Black Holes Radiate Mainly on the Brane, Phys. Rev. Lett. 85, 499 (2000).

[52] S. Dimopoulos and G. L. Landsberg, Black Holes at the LHC, Phys. Rev. Lett. 87, 161602 (2001).

[53] M. Kachelriess and M. Plumacher, Ultrahigh-energy neutrino interactions and weak scale string theories, Phys. Rev. D 62, 103006 (2000).

[54] L. Anchordoqui, H. Goldberg, T. McCauley, T. C. Paul, S. Reucroft, and J. Swain, Extensive air showers with $\mathrm{TeV}$ scale quantum gravity, Phys. Rev. D 63, 124009 (2001).

[55] A. Kappes, Multi-PMT optical module designs for IceCubeGen2, EPJ Web Conf. 116, 01001 (2016).

[56] M. Ackermann (IceCube Gen2 Collaboration), The IceCube neutrino observatory contributions to ICRC 2017 Part VI: IceCube-Gen2, the next generation neutrino observatory, arXiv: 1710.01207.

[57] L. Classen et al. (IceCube Collaboration), A multi-PMT optical module for the IceCube upgrade, arXiv:1908.10802.

[58] J. L. Feng, P. Fisher, F. Wilczek, and T. M. Yu, Observability of Earth Skimming Ultrahigh-Energy Neutrinos, Phys. Rev. Lett. 88, 161102 (2002).

[59] A. Schneider et al. (IceCube Collaboration), Characterization of the astrophysical diffuse neutrino flux with IceCube high-energy starting events, arXiv:1907.11266. 